\title{
ARTICLE
}

\section{Dielectric thermometer by using the quantum paraelectricity for microcalorimeter}

\author{
Yasuhiro IKEDA ${ }^{1 *}$, Yoshiki NAGAFUCHI ${ }^{1}$, Keisuke MAEHATA $^{1}$, Kenji ISHIBASHI $^{1}$, Takahiro UMENO ${ }^{2}$, \\ Hiroshi TAKASHIMA ${ }^{3}$ and Bambang PRIJAMBOEDI ${ }^{3}$ \\ ${ }^{1}$ Department of Applied Quantum Physics and Nuclear Engineering, Kyushu University \\ 744 Motooka, Nishi-ku, Fukuoka 819-0395 Japan \\ 2 Taiyo Nippon Sanso Corporation, Tsukuba, Japan \\ ${ }^{3}$ National Institute of Advanced Industrial Science and Technology \\ 1-1-1 Umezono, Tsukuba, Ibaraki 305-8568, Japan
}

\begin{abstract}
The electric capacitance of a $\mathrm{SrTiO}_{3}$ thin film was measured at frequencies from 1 to $100 \mathrm{kHz}$ by using an impedance analyzer with four-terminal method in the temperature range from 100 to $200 \mathrm{mK}$. The values of the electric capacitance exhibited large temperature dependence below temperature of $110 \mathrm{mK}$. The temperature dependence of the electric capacitance is expected to be utilized for the dielectric thermometers of the microcalorimeter.
\end{abstract}

KEYWORDS: microcalorimeter, $\mathrm{SrTiO}_{3}$ thin film, the dielectric thermometer, the temperature dependence of the dielectric constant

\section{Introduction}

A microcalorimeter is a radiation particle detector indicating the energy of incident particle by measuring a rise in temperature of an absorber. A sensitive thermometer is an important component of the microcalorimeter. Various types of thermometers for the microcaloriemeter have been developed and exhibited excellent energy resolution below $10 \mathrm{eV}$ of FWHM in X-ray detection ${ }^{1)}$.

A dielectric thermometer has been proposed for microcalorimeter utilizing the dependence of the dielectric constant on temperature ${ }^{2)}$. The temperature rise induced by the energy deposition of the incident radiation is converted into electric charge proportional to the change in dielectric constant of the dielectric thermometer.

$\mathrm{A} \mathrm{SrTiO}_{3}$ (STO) thin film was developed for a cryogenic thermometer ${ }^{3)}$. The STO thin film has a potential of a sensitive thermometer for the microcalorimeter. In this work, the electric capacitances and the loss factors of the STO thin film were measured at frequencies from 1 to $100 \mathrm{kHz}$ in the temperature range from 100 to $200 \mathrm{mK}$.

\section{Dielectric microcalorimeter}

Figure 1 shows the schematic drawing of the operating concept of the dielectric microcalorimeter with the heat capacitance $C_{V}$ and the electric capacitance $C_{d}$. The thermal link with the conductance $G$ connects the dielectric calorimeter to the cold stage maintained at the base temperature $T_{0}$. The temperature of the dielectric calorimeter is raised by the energy deposition of the incident particle, and falls down to $T_{0}$ with time constant of thermal relaxation $\tau=C_{V} / G$. The transient temperature change of the dielectric calorimeter by absorbing the energy $E$ of the incident particle is expressed by

$$
\Delta T(t)=T(t)-T_{0}=\frac{E}{C_{V}} \exp \left(-\frac{t}{\tau}\right) .
$$

\footnotetext{
${ }^{*}$ Corresponding author, E-mail: number4410ne@kune2a.nucl.kyushuu.ac.jp

(c)Atomic Energy Society of Japan
}

The electric capacitance $C_{d}$ of the dielectric calorimeter changes with the temperature $T(t)$. The electric charge $Q$, stored in the dielectric material by applying a constant DC voltage $V_{B}$, alters with changing of $C_{d}$. Therefore the energy $E$ of the incident particle is converted into a change in the electric charge, given as

$$
\begin{aligned}
\Delta Q=V_{B} \Delta C_{d} & =V_{B}\left(\frac{d C_{d}}{d T}\right) \Delta T \\
& =\frac{E V_{B}}{C_{V}}\left(\frac{d C_{d}}{d T}\right) \exp \left(-\frac{t}{\tau}\right) .
\end{aligned}
$$

To generate a pulse signal from the detector, $\Delta Q$ is collected by the charge sensitive preamplifier. The feedbackresistance and capacitance of the preamplifier are $R_{f}$ and $C_{f}$, respectively. The time constant of the preamplifier is assumed to be $C_{f} R_{f} \gg \tau$. Finally, the output voltage signal of the incident particle detection by the dielectric microcalorimeter is given by

$$
\begin{aligned}
V_{\text {out }} & =\frac{\Delta Q}{C_{f}} \\
& =\frac{E V_{B}}{C_{f} C_{V}}\left(\frac{d \ln C_{d}}{d T}\right) C_{d}\left(T_{0}\right) \exp \left(-\frac{t}{\tau}\right) .
\end{aligned}
$$

The energy solution $\Delta E$ of the dielectric microcalorimeter is defined by ${ }^{4}$

$$
\Delta E(F W H M)=2.35 \sqrt{k_{B} C_{V} T_{0}^{2} / \alpha},
$$

where $k_{B}$ is Boltzmann constant and $\alpha$ is the sensitivity of the dielectric microcalorimeter expressed by

$$
\alpha=\frac{d\left(\ln C_{d}\right)}{d(\ln T)} .
$$

From eqs. (4) and (5), the energy resolution of the dielectric microcalorimeter improves with increasing the value of $\alpha$.

The dielectric microcalorimeter has advantages of suppressing the Johnson noise and the Joule heat generation in the device. 


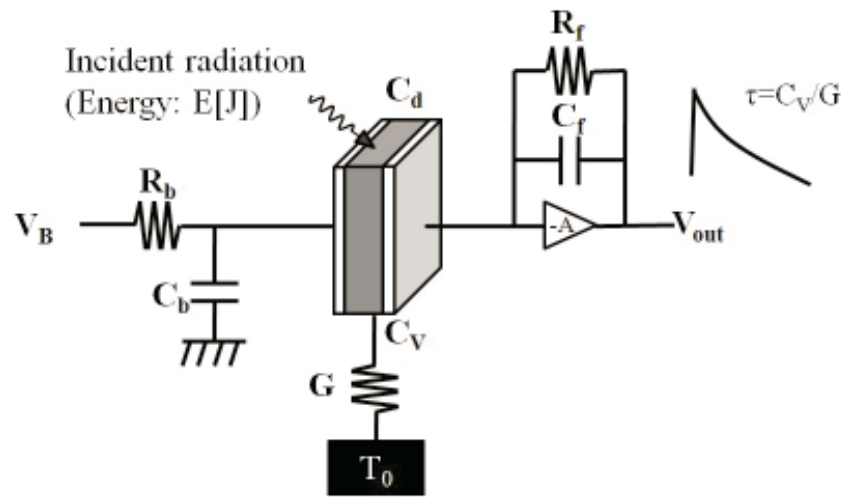

Fig. 1 Operating concept of the dierectric microcalorimeter. $\left(V_{B}\right.$ : a constant DC voltage applied, $R_{b}$ and $C_{b}$ : low pass filter, $C_{d}$ : electric capacitance, $C_{V}$ : heat capacitance, $G$ : heat conductance, $T_{0}$ : operating temperature, $R_{f}$ and $C_{f}$ : feedbackresistance and capacitance of the preamplifier, $V_{\text {out }}$ : output voltage signal, $\tau$ : time constant)

\section{The epitaxially grown STO thin film}

The STO is a typical quantum paraelectric material. The dielectric constant of the quantum paraelectric materials increases with cooling down. However the quantum paraelectric materials do not undergo ferroelectric transition at low temperatures because of quantum fluctuation, but holds the high values of the dielectric constant $t^{5)}$. A certain kind of the quantum paraelectric materials was found to exhibit the temperature dependence of the dielectric constant around $100 \mathrm{mK}^{6}$.

A STO thin film was developed for the capacitance cryogenic thermometer at National Institute of Advanced Science and Technology ${ }^{3}$. The STO thin film has a potential of a sensitive thermometer for the microcalorimeter. The capacitance thermometer consists of epitaxially grown STO thin film between two $\mathrm{YBa}_{2} \mathrm{Cu}_{3} \mathrm{O}_{7}{ }_{\delta}(\mathrm{YBCO})$ electrodes.

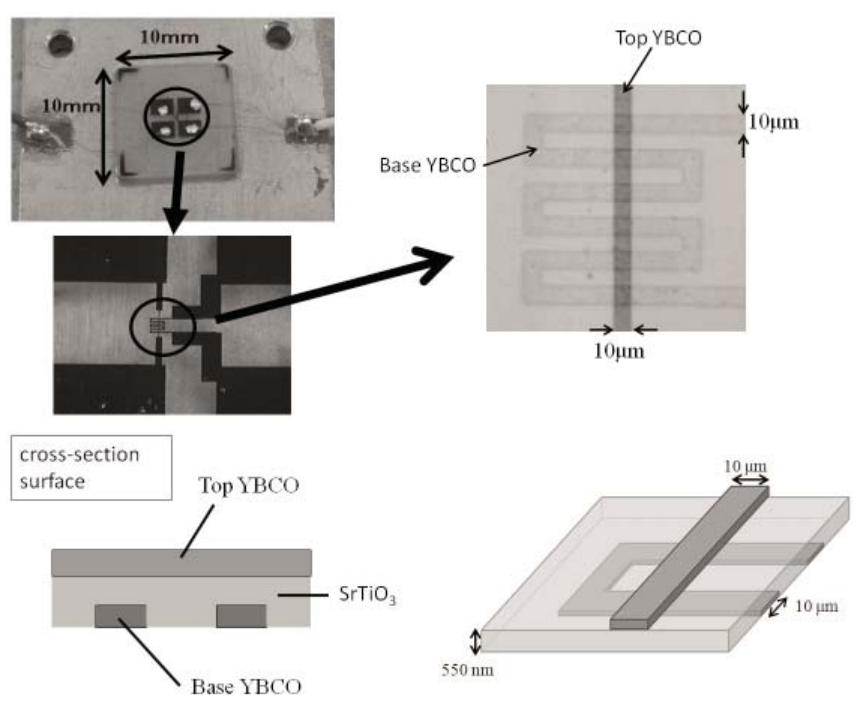

Fig. 2 The fabricated epitaxially grown $\mathrm{YBCO} / \mathrm{STO} / \mathrm{YBCO}$
Figure 2 shows photographs of the fabricated epitaxially grown $\mathrm{YBCO} / \mathrm{STO} / \mathrm{YBCO}$. The epitaxially grown STO thin film of $550 \mathrm{~nm}$ thick was deposited on the meandering shaped line $10 \mu \mathrm{m}$ wide of the YBCO base electrode. As shown in Fig. 2, six parallel capacitors are produced at intersections between the top and the base electrodes. The electric capacitance at $300 \mathrm{~K}$ was $14 \mathrm{pF}$.

A liquid-helium-free ${ }^{3} \mathrm{He}-{ }^{4} \mathrm{He}$ dilution refrigerator was employed to cool the dielectric thermometer. The liquid-heliumfree ${ }^{3} \mathrm{He}-{ }^{4} \mathrm{He}$ dilution refrigerator was manufactured by Taiyo Nippon Sanso Corporation. A schematic drawing of the liquid-helium-free ${ }^{3} \mathrm{He}-{ }^{4} \mathrm{He}$ dilution refrigerator is illustrated in Fig. 3.

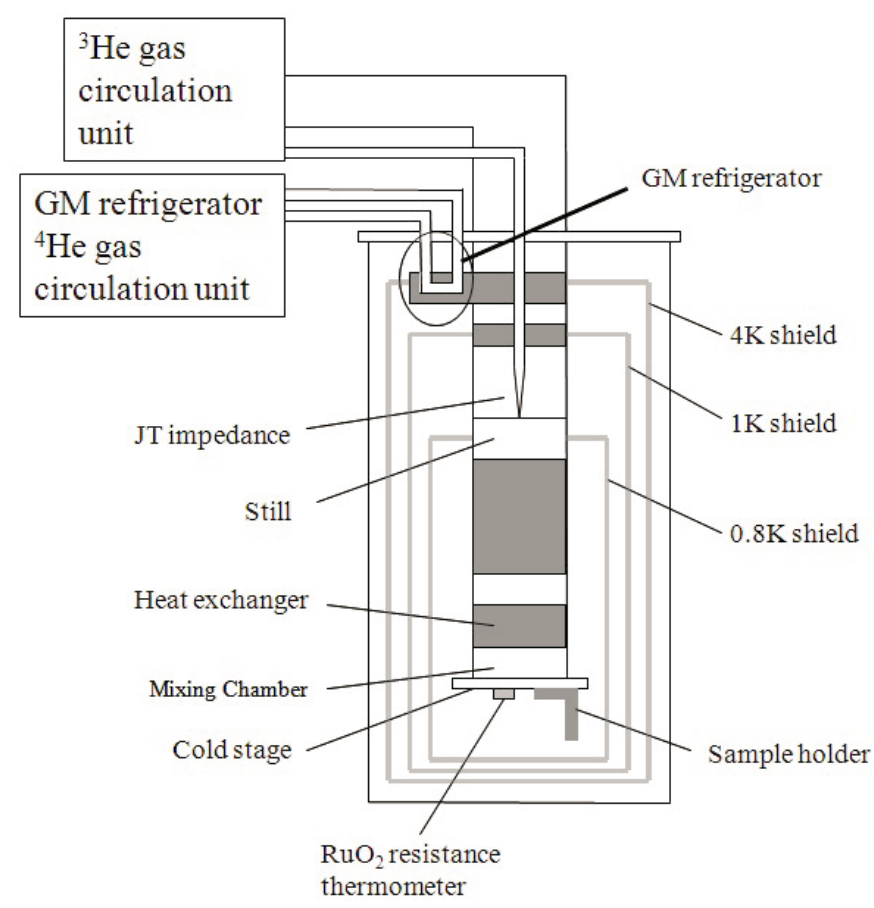

Fig. 3 Schematic drawing of the liquid-helium-free $3 \mathrm{He}-$ 4He dilution refrigerator

The ${ }^{3} \mathrm{He}-{ }^{4} \mathrm{He}$ dilution refrigerator is operated without consuming liquid helium by loading a Gifford-McMahon (GM) cooler.

The STO thin film chip was glued on the copper plate with the GE7031 varnish. The copper plate was placed on the holder bolted to the cold stage of the refrigerator.

\section{The temperature dependence of dielectric con- stant at various frequencies}

The electric capacitance and the dissipation factor of the STO thin film were measured at frequencies from 1 to $100 \mathrm{kHz}$ by using an impedance-analyzer with four-terminal method in the temperature range from 100 to $200 \mathrm{mK}$. Figure 4 shows relationship between obtained real values of the electric capacitance and the temperature. Since the value of the electric capacitance of the STO thin film was measured to be $14 \mathrm{pF}$ at $300 \mathrm{~K}$, the quantum paraelectricity of the STO thin film was confirmed by a growth in the electric capacitance. Due to 


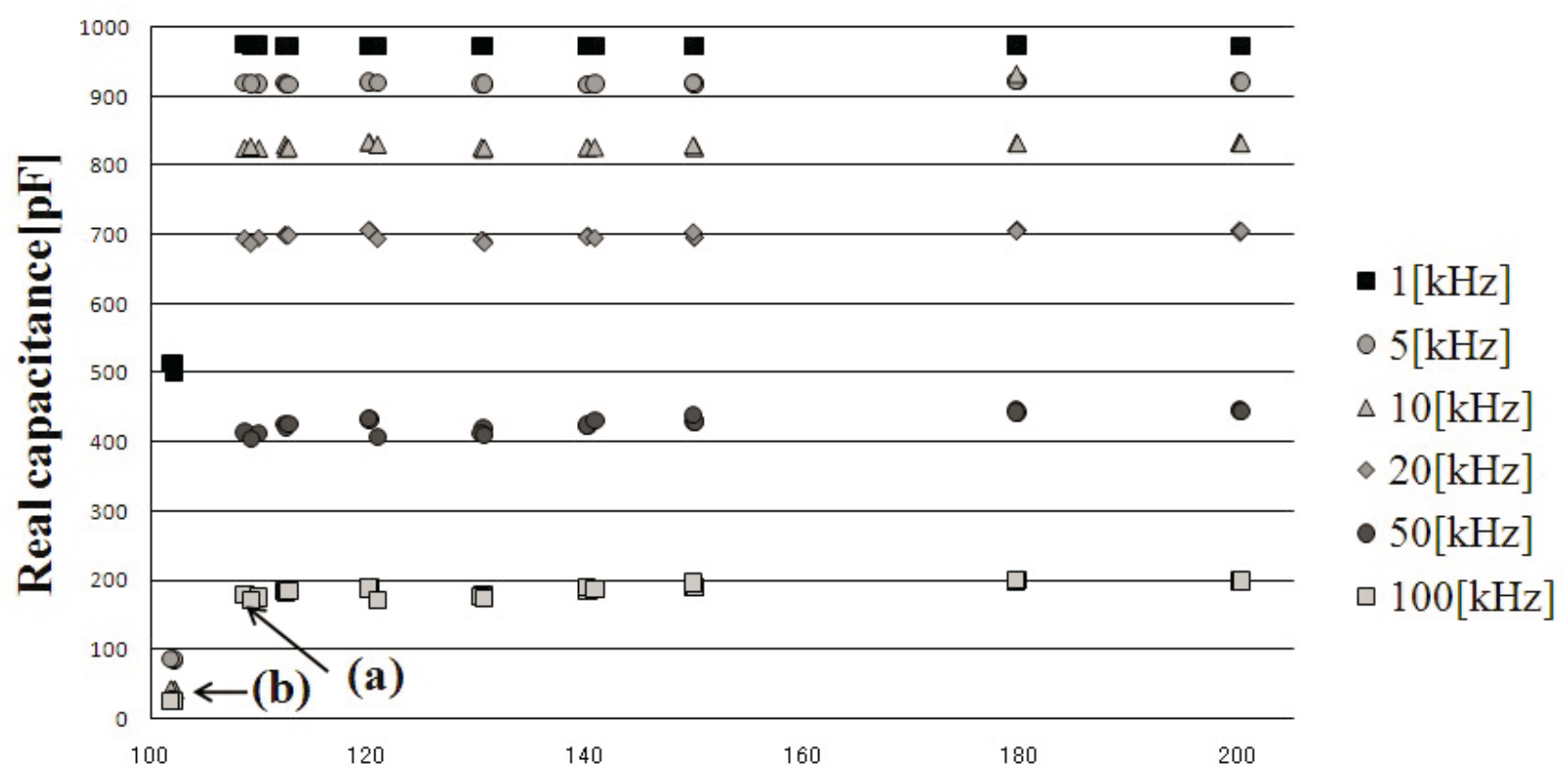

Temperature[mK]

Fig. 4 Variation of the real electric capacitance as a function of temperature. ((a): $108.4 \mathrm{mK}, 178.9 \mathrm{pF}, 100 \mathrm{kHz},(\mathrm{b}): 102.0 \mathrm{mK}$, $25.1 \mathrm{pF}, 100 \mathrm{kHz}$. The sensitivity of the thermal sensor $\alpha$ is $28.7 \pm 4.1$.)

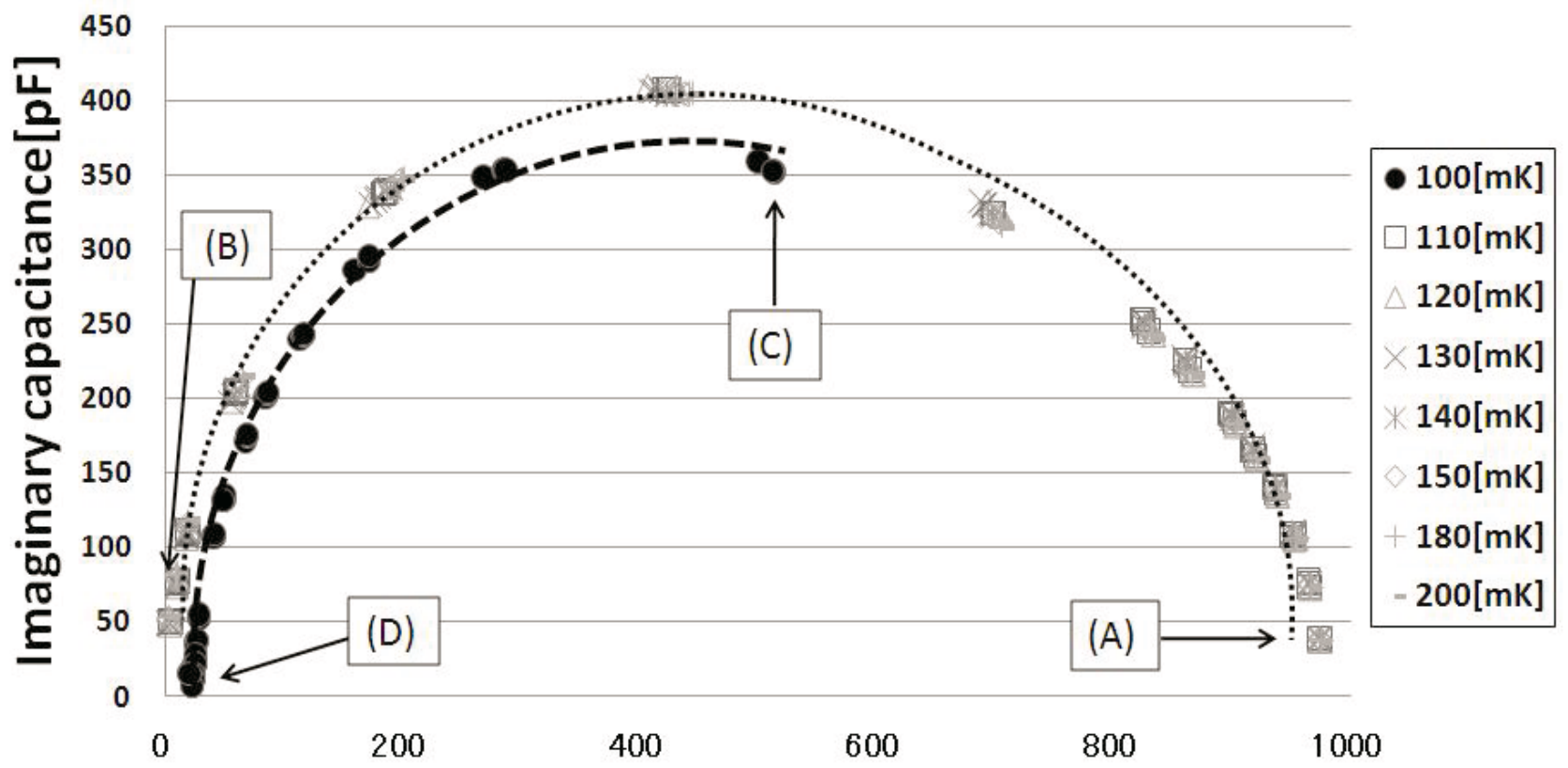

Real capacitance[pF]

Fig. 5 Relationship between the imaginary and real components of electric capacitance. ((A): 110-200 mK, $1 \mathrm{kHz},(\mathrm{B})$ : 110 $200 \mathrm{mK}, 100 \mathrm{kHz},(\mathrm{C}): 100 \mathrm{mK}, 1 \mathrm{kHz},(\mathrm{D}): 100 \mathrm{mK}, 100 \mathrm{kHz}$.)

the quantum paraelectricity the electric capacitance holds constant values in the temperature range from $110 \mathrm{mK}$ to $200 \mathrm{mK}$. At temperatures below $110 \mathrm{mK}$, values of the electric capacitance decrease with temperature. A change in values of the electric capacitance implies a violation of the quantum paraelectricity.

In Fig. 4, (a) are three points at temperature $110 \mathrm{mK}$ and

(b) are three points at temperature $100 \mathrm{mK}$, at the frequency 
$100 \mathrm{kHz}$. These six points were converted into the double logarithm, and the inclination, i.e., the sensitivity for the dielectric microcalorimeter $\alpha$ was calculated by the least squares method. The sensitivity $\alpha$ is estimated to be $28.7 \pm 4.1$ at frequency of $100 \mathrm{kHz}$. The energy resolution of the dielectric microcalorimeter is expected to be $3.3 \pm 0.2 \mathrm{eV}$ of FWHM value with assuming the value of the heat capacitance of the dielectric thermometer to be $10^{11} \mathrm{~J} / \mathrm{K}$.

Complex electric capacitance was obtained from experimental values of the real electric capacitance and the dissipation factor. Figure $\mathbf{5}$ shows relationship between the imaginary and real components of electric capacitance. Real components indicate the actual electric capacitance, while imaginary components correspond to the product of the actual electric capacitance and the dissipation factor.

In Fig. 5, the point (A) was obtained at the frequency of $1 \mathrm{~Hz}$ in the temperature range from 110 to $200 \mathrm{mK}$. With increasing frequency, experimental points moved counterclockwise from the point (A) along the circular arc and arrived at the point (B) at the frequency of $100 \mathrm{kHz}$. On the other hand the point $(\mathrm{C})$ was obtained at the frequency of $1 \mathrm{kHz}$ at a temperature of $100 \mathrm{mK}$. With increasing frequency, experimental points moved counterclockwise from $(\mathrm{C})$ and arrived at the point (D) at a frequency of $100 \mathrm{kHz}$. Change in trajectories in Fig. 5 would imply a break in the quantum paraelectricity in the temperature region from 100 to $110 \mathrm{mK}$.

\section{Conclusion}

The electric capacitance of the epitaxially grown $\mathrm{SrTiO}_{3}$ thin film was measured at frequencies from 1 to $100 \mathrm{kHz}$ by using an impedance-analyzer with four-terminal method in the temperature range from 100 to $200 \mathrm{mK}$. The $\mathrm{SrTiO}_{3}$ thin film exhibited the quantum paraelectricity in temperature range from $110 \mathrm{mK}$ to $200 \mathrm{mK}$. The electric capacitance was found to decrease with temperatures below $110 \mathrm{mK}$. The sensitivity $\alpha$ of the dielectric microcalorimeter was evaluated to be $28.7 \pm 4.1$ at frequency $100 \mathrm{kHz}$. The energy resolution of the dielectric microcalorimeter expected $3.3 \pm 0.2 \mathrm{eV}$ of FWHM value assuming the value of the heat capacitance of the dielectric thermometer to be $10^{11} \mathrm{~J} / \mathrm{K}$.

Frequency characteristics of the electric capacitance was found to change in profile of obtained relationship between the imaginary and real components of electric capacitance at a temperature of $100 \mathrm{mK}$.

\section{Acknowledgment}

Authors acknowledge T. Yasumune, H. Ogi, T. Masuda, Y. Ooshima, M. Maeda, for their support on experiments.

\section{References}

1) J. N. Ullom, J. A. Baell, W. B. Doriese, "Optimized transitionedge x-ray microcalorimeter with $2.4 \mathrm{eV}$ energy resolution at $5.9 \mathrm{keV}, "$ APPLIED PHYSICS LETTERS 87, 194103 (2005)

2) W. N. Lawless, J. Opt. Soc. Am., 62, 1449 (1972)

3) H. Takashima, Ruiping Wang, N. Shirakawa, et al., "Capacitance temperature sensor using epitaxial $\mathrm{SrTiO} 3$ film with a single-crystal-like behavior," Thin Solid Films, 486, 145 (2005)

4) Ch. Enss (Ed), Cryogenic Particle Detection, Topics in Applied Physics, 99, Springer-Verlag Berlin Heidelberg (2005)

5) K. A. Müller, H. Burkard, Phys. Rev. B, 19, 3593(1979)

6) W. N. Lawless, R. Radebaugh, R. J. Soulen, Rev. Sci. Instr., 42, 567 (1971) 